\title{
UMA ANÁLISE DO CONCEITO DE INTELIGÊNCIA ATRAVÉS DA NOÇÃO DE MODELO NA CIÊNCIA COGNITIVA
}

\section{AN ANAL YSIS OF THE CONCEPT OF INTELLIGENCE THROUGH THE NOTION OF MODEL IN COGNITIVE SCIENCE}

\author{
Nathália Cristina Alves Pantaleão *
}

Recebido: 09/2016

Aprovado: 10/2016

\begin{abstract}
Resumo: O objetivo do presente trabalho é analisar em que medida um modelo pode ser uma ferramenta explicativa para a abordagem do conceito de inteligência. Para tal, nos deteremos em um primeiro momento, na tentativa de clarificar os conceitos de modelo e de inteligência. Em seguida traremos, à baila, o projeto mecanicista de modelagem da mente proposto pela Inteligência Artificial (IA), enquanto um ramo da Ciência Cognitiva. Por fim, argumentaremos que a proposta de modelagem da inteligência desenvolvida pela Ciência Cognitiva tradicional não é suficiente e nesse sentido, apontaremos para a hipótese, segundo a qual, o conceito de inteligência poderia ser modelado com maior plausibilidade a partir de modelos explicativos sistêmicos.
\end{abstract}

Palavras-Chave: inteligência, modelo,representação, sistêmica.

\begin{abstract}
The objective of this paper is to analyze the extent to which a model can be an explanatory tool to approach the concept of intelligence. For this purpose we will focus, at first, in trying to clarify the concepts of model and intelligence. Then we introduce the mechanistic modeling project of the mind proposed by Artificial Intelligence (Al), while a branch of the Cognitive Science. Finally, we argue that the proposed model of intelligence developed by traditional cognitive science is not enough, and accordingly, will point to the hypothesis, according to which the concept of intelligence could be modeled with greater plausibility from systemic explanatory models.
\end{abstract}

Keywords: Intelligence, Model, Representation, Sistemic

\section{Introdução}

Neste trabalho, pretendemos analisar o alcance explicativo dos modelos na Ciência Cognitiva. Para isso, analisaremos os pressupostos internalistarepresentacionistas da tradição filosófica em relação à explicação do comportamento inteligente e sua possível extensão na Inteligência Artificial tradicional (IA). No que se refere aos objetivos da IA, a maioria dos

\footnotetext{
* Doutoranda em Filosofia, UNICAMP.

Problemata: R. Intern. Fil. v. 7. n. 2 (2016), p. 241-253 ISSN 2236-8612 doi:http://dx.doi.org/10.7443/problemata.v7i2. 29977
} 
pesquisadores (TURING, 1950; MINSKY, 1991, 1995) concorda quando se afirma que seu projeto de pesquisa pretende desenvolver programas computacionais que, em principio, possam se "comportar" ou "pensar" de maneira inteligente. Para isso, inicialmente os pesquisadores procuraram estabelecer algumas definições básicas de conceitos, como o de programa, algoritmo e o próprio conceito de inteligência, e técnicas de desenvolvimento de modelos computacionais. Este programa de pesquisa busca construir modelos para simular, e talvez emular, processos inteligentes, sendo que o funcionamento do modelo constituiria um teste de uma teoria específica sobre o processo por ele simulado. Seria, justamente, a partir desse ponto que o consenso entre os pesquisadores da IA diminui. Isso se deve a que algumas definições enfatizam a criação de programas, outras se concentram em linguagens de programação a fim de simular os processos de pensamento, enquanto outros pesquisadores consideram relevante qualquer programa que cause consequências inteligentes, mesmo que sejam no nível da simulação. Tendo em vista tal problemática da IA, estruturamos o presente trabalho como se segue.

Inicialmente, apresentaremos os conceitos de modelo-modelagem e de inteligência na Ciência Cognitiva, posteriormente traremos à baila um modelo clássico na Ciência Cognitiva, que busca modelar a inteligência a partir da resolução de problemas. Por fim, a partir dessa explanação, problematizaremos os limites e os alcances do modelo enquanto ferramenta explicativa.

\section{O conceito de modelagem e de inteligência na Ciência Cognitiva}

O ramo da Ciência Cognitiva denominado Inteligência Artificial surgiu do projeto de pesquisadores de diversas áreas de entender e reproduzir comportamentos humanos considerados inteligentes. Mas em que consiste a inteligência? O que se quer dizer quando se diz que alguém (ou um sistema) é inteligente? Como apontamos na seção anterior, para os primeiros pesquisadores da IA, a inteligência consistia na resolução satisfatória de problemas. Nesse sentido, é inteligente o ser capaz de manipular e empregar certos conhecimentos (regras, teoremas, etc.) de modo a alcançar uma solução satisfatória de um problema a partir de um conjunto de soluções possíveis. Todavia, essa é apenas uma parcela da amplitude do conceito de inteligência, que ainda não está bem definido. Como aponta Murphy (2000, p. 15, tradução nossa): 
[A] Inteligência Artificial geralmente não tem definições comumente aceitas. Um dos primeiros textos da IA defini-a como 'o estudo de ideias que possibilitem computadores inteligentes', o que parecia evitar a questão [o que é ser inteligente?]. Um texto recente é mais específico, 'IA é a tentativa de que o computador faça coisas que, no momento, pessoas fazem melhor'. Esta definição é interessante porque implica que, sendo a tarefa realizada com sucesso por um computador, então a técnica que tornou isso possível já não é mais IA, mas algo mundano [do mundo]. ${ }^{1}$

Podemos apontar que o conceito de inteligência também diz respeito ao plano da ação e não só ao da manipulação simbólica, cuja relevância foi maximizada pelos primeiros cientistas cognitivos. A satisfatoriedade com a qual os problemas são resolvidos diz respeito à resolução natural e humana que serve de modelo para a representação de tais problemas. Nesse sentido, o paradigma da inteligência adotado pela IA focaliza e privilegia certas capacidades de seres humanos e tais capacidades servem de modelo para a construção de sistemas artificiais. $O$ que vale ser ressaltado aqui é que os pesquisadores da IA mantiveram (e mantêm) a característica inteligência no processo de abstração para a construção de modelos.

Assim, podemos dizer que o método de modelagem adotado pelos pesquisadores da IA revela suas concepções pessoais: por que dentre tantas características humanas privilegiaram a inteligência no processo de modelagem computacional? A resposta a esta questão diz respeito à hipótese de que a inteligência, ao ser considerada um processo de manipulação simbólica a fim de solucionar um problema, seria a capacidade humana mais relevante e com maior plausibilidade de modelagem. Podemos dizer que tal hipótese se fundamenta nas técnicas de programação lógica e mecanização física vigente, pois estas impossibilitam, em certo sentido, a modelagem de outros tipos de características humanas, como as relacionadas a emoções, por exemplo, e porque os cientistas cognitivos consideram, efetivamente, a inteligência simbólica a expressão específica da condição humana.

A postura de que a inteligência diz respeito à manipulação simbólica permeia não apenas o ramo da Ciência Cognitiva, mas também outros ramos das Ciências Humanas, como a Psicologia e a Filosofia da Linguagem. Vemos que tal postura parece perpetuar e influenciar a maioria dos sistemas explicativos em relação ao conceito de inteligência. Nesse sentido, o psicólogo Robert Sterbenberg (1986, p. 51) afirma que: "[...] a inteligência envolve a capacidade de solucionar problemas abstratos". Esta caracterização da inteligência se estende ao projeto de modelagem computacional da Ciência Cognitiva, mas suas raízes remetem aos pensamentos dos racionalistas como René Descartes. Segundo Descartes, a inteligência é uma característica que diz respeito à mente enquanto matéria pensante (res cogitans). Nesse sentido, a natureza corpórea (res extensa) está submetida a leis físicas, mas estaria, 
segundo Descartes, causalmente relacionada à mente. Nas palavras do filósofo: "Considerava-me, inicialmente, como provido de rosto, mãos, braços e toda essa máquina composta de ossos e carnes, tal como um cadáver, a qual eu designava corpo" (DESCARTES, 1988, p. 25). Por outro lado, a mente não se sujeitaria, para Descartes, às restrições da corporeidade, pois não se encontra no espaço, suas funções não estão sujeitas a leis mecânicas e não são observáveis: seu desenvolvimento é privado e se caracteriza por uma experiência individual e introspectiva. A mente seria "algo extremamente raro e sutil, como um vento, uma flama ou um ar muito tênue" que insinua o movimento do corpo e torna-se responsável pelo pensamento. Em relação ao pensamento, Descartes (1988, p. 25-26) afirma que:

\begin{abstract}
[...] é um atributo que me pertence; só ele não pode ser separado de mim. Eu sou, eu existo: isto é certo; mas por quanto tempo? A saber, por todo o tempo em que penso; pois poderia, talvez, ocorrer que, se eu deixasse de pensar, deixaria ao mesmo tempo de ser ou de existir. Nada admito agora que não seja necessariamente verdadeiro: nada sou, pois, falando precisamente, senão uma coisa que pensa, isto é, um espírito, um entendimento ou uma razão, que são termos cuja significação me era anteriormente desconhecida.
\end{abstract}

A essência do ser humano é assentada na faculdade de pensar e esta por sua vez, é fundamentada metafisicamente por vias racionais. Em outro momento Descartes deixa claro como entende a natureza do ser: "compreendi por aí que era uma substância cuja essência ou natureza consiste apenas no pensar, e que, não necessita de nenhum lugar, nem depende de qualquer coisa material" (DESCARTES, 1987, p. 47). Assim sendo, o pensamento constitui a essência do ser na medida em que o torna capaz de atribuir sentido às coisas do mundo através da reflexão interna. Tal como apontado por Khalfa (1996, p. 7-8), "Os 'dualistas' postulavam que a inteligência era uma faculdade exclusiva dos seres regrados por uma substância imaterial, um espírito, do qual uma das mais claras manifestações no mundo era o comando da fala [...]". Pelo viés dualista substancial, a inteligência não diria respeito a um substrato físico, mas imaterial ou mental relacionado à capacidade de representar e de manipular regras lógicas, tese com a qual concordam, grosso modo, os cientistas cognitivos. Entretanto, os cientistas cognitivos contemporâneos defendem a tese de que comportamentos inteligentes podem ser simulados por sistemas artificiais, como fazem, por exemplo, Newell; Shaw \& Simon (1963), Putnam (1967/1975) e Minsky (1991) e, assim sendo, a inteligência não mais seria uma propriedade exclusiva dos seres humanos.

Como já observamos, embora o conceito de inteligência seja de grande importância na Ciência Cognitiva, não se trata de um conceito bem definido. Nesse sentido, a construção de modelos se apresenta como também método e ferramenta explicativa de conceitos nebulosos como o de inteligência. Trata-se 
da tentativa de explicar processos complexos através de mecanismos fundamentados na demonstração e na dedução. Desse modo, por meio do funcionamento do modelo, o próprio conceito em questão se tornaria, em certo sentido, auto-evidente. A partir da construção de modelos, o ser humano conhece e se reconhece. Mas o que é um modelo?

Inicialmente o modelo diz respeito à construção de mecanismos artificiais de objetos e características que, dada a relevância, merecem ser imitadas (ou, no caso, modeladas). O modelo, em certo sentido, possibilita que um recorte da realidade, de acordo com um critério de relevância, seja controlado e suas variáveis minimamente previstas, mesmo que seu comportamento não esteja contido inicialmente no modelo. Nas palavras de Dupuy (1996, p. 24), "O modelo abstrai da realidade fenomenal o sistema das relações funcionais consideradas por ele as únicas pertinentes, pondo, por assim dizer, entre parênteses tudo o que não depende desse sistema [...]". Desse modo, por ser a representação de uma parte específica e delimitada de fenômenos, muitas vezes o modelo acaba se distanciando da realidade. Em outras palavras, por não conter a representação da totalidade dos fenômenos da realidade, o modelo se emancipa, em certo sentido, dessa realidade, embora ainda contenha em si as representações básicas que o tornam um modelo.

O modelo apresenta-se como uma ferramenta de controle explicativo e preditivo de maior eficiência, rapidez e elegância que o mundo fenomenal não possui. Em certo sentido, o ser humano conhece quando consegue organizar o mundo fenomenal. O modelo é uma estrutura de representação de um objeto ou, mais especificamente, de um recorte do mundo fenomenal que serve de explicação para um fenômeno específico. Como aponta Minsky (1995, p. 1) "Nós usamos o termo modelo nesse sentido: Para um observador $B$, um objeto $A^{*}$ é um modelo de um objeto $A$ na medida em que $B$ pode utilizar $A^{*}$ para responder questões que lhe interessam sobre $A{ }^{2}$ ".

Quando se trata de um modelo computacional, consideramos que esse tipo de modelo é uma estrutura lógica de representação de um processo ou capacidade cognitiva de um sistema, cuja eficácia se revela através do desempenho de uma função computável. Uma função é computável quando existem regras definidas e explícitas que, ao serem seguidas, são capazes de computar o valor para quaisquer argumentos dados (BOOLOS et al, 2012). Alan Turing foi o responsável por desenvolver a máquina teórica que acabou por formalizar a noção de computação e, consequentemente, a de algoritmo, conceitos chave para o desenvolvimento dos modelos da IA. Posteriormente, pesquisadores da Ciência Cognitiva tratavam a inteligência em termos da execução de funções específicas e resoluções de problemas. Analisaremos um desses projetos, a seguir. 


\section{O Solucionador Geral de Problemas: a medida da inteligência em termos de resolução de problemas}

Para a maioria dos cientistas cognitivos, os modelos computacionais que seriam capazes de realizar satisfatoriamente uma função específica, seriam necessariamente e essencialmente capazes de resolver um problema. Assim, em 1959, Newell, Shaw e Simon criam o "Solucionador Geral de Problemas (GPS) 3", um programa de computador destinado à resolução de qualquer tipo de problema passível de representação simbólica, tais como resolver e provar teoremas matemáticos, jogar xadrez ou até entender o significado de um texto literário o qual, segundo os autores, não passaria do resultado de um processo de manipulação simbólica e sintática (NEWELL; SHAW \& SIMON, 1963, p. 109). O objetivo consistia, então, em, através do GPS, entender o processo complexo que efetivamente tange à resolução do problema em questão. A estratégia dos pesquisadores nos estudos desses sistemas complexos era "[...] especificar, em detalhes, programas para computadores digitais e estudar seu comportamento empiricamente, rodando os programas com variações e sob uma variedade de condições " (NEWELL; SHAW \& SIMON, 1963, p. 110, tradução nossa).

Entretanto, o GPS foi construído a partir de um paradigma mecânico e procedural (tal como fez Babbage), cuja funcionalidade estava contida em instruções representadas por meio de marcas em papéis ou cartões perfurados. Tratava-se, então, de um programa puramente formal baseado em regras lógicas, mais precisamente, em cálculos sentenciais. Nas palavras dos autores:

\footnotetext{
O cálculo sentencial é um sistema matemático formalizado, consistindo de expressões construídas a partir de combinações de símbolos básicos. Cinco dessas expressões consideradas axiomas e há regras de inferência para a geração de novos teoremas a partir dos axiomas e de outros teoremas (Newell, Shaw e Simon, 1963, p. 110, tradução nossa) ${ }^{5} 6$
}

Os processos realizados pelo programa limitavam-se, exclusivamente, ao âmbito da matemática formal, e sendo assim, não necessitavam de algum tipo de interpretação. O comportamento do programa de Newell, Shaw e Simon era baseado nos conceitos de problema, algoritmo e heurística. Para os autores, problema é tudo aquilo que é passível de ser resolvido: alguém teria um problema apenas se pudesse selecionar sua solução entre um conjunto de possibilidades (NEWELL, SHAW \& SIMON, 1963, p. 113). Partindo do pressuposto de que para todo problema existe uma solução, dado um problema específico, o GPS necessariamente deveria encontrar uma maneira de solucioná-lo. Para Newell, Shaw e Simon a solução para problemas dados 
consistiria em encontrar algoritmos e uma heurística apropriados: algoritmo enquanto um processo delimitado e especificado a partir de regras não ambíguas, e heurística enquanto um método simplificador que contém em si operações que resolvem o problema (NEWELL, SHAW \& SIMON, 1963).

Em suma, a partir de um problema dado, o GPS testa um método (constituído por axiomas e teoremas pré-estabelecidos e armazenados em uma lista de teoremas), a fim de solucioná-lo, assim como exclui os métodos mal sucedidos para solucionar o problema em questão. Se o método testado falha, o problema é fragmentado em subproblemas, ou seja, em problemas parciais cuja probabilidade de resolução seria maior devido à amplitude de sua constituição. Estes subproblemas eram adicionados a uma lista de subproblemas. Se todas as tentativas falham, ou seja, nenhum teorema da lista de teoremas resolve o problema ou o subproblema, a mesma rotina se repete normalmente e depois se retroalimentando. Primeiro tenta-se criar um subproblema, em seguida tenta-se resolvê-lo através de um método, se esta tentativa falha, coloca-se o subproblema na lista e como apontam Newell, Shaw e Simon (1963, p. 119, tradução nossa): "Pela natureza dos métodos, se o método de substituição é sempre bem sucedido para cada um dos subproblemas, o teorema original é provado ${ }^{7 "}$. Por fim, se todos os métodos foram testados e o problema não foi solucionado, o programa seleciona 0 próximo subproblema e executa os mesmos passos descritos anteriormente.

O que nos cabe ressaltar aqui é que Newell, Shaw e Simon entendiam que o desenvolvimento do GPS representava, de fato, o pensamento humano. Para eles o pensar equivaleria à manipulação de axiomas e teoremas a fim de resolver um problema artificial, no sentido de que tal problema não é legitimamente um problema do programa, mas sim um dado externo embutido. Além do mais, tradicionalmente, os problemas a serem resolvidos por este tipo de programa consistiam em operações matemáticas ou lógicas, embora seus desenvolvedores afirmem que seria um programa para a solução de qualquer tipo de problema. Essa postura revela, ainda, a presença de pressupostos internalistas, racionalistas e simbólicos desvinculados do plano da ação.

O que podemos ressaltar aqui, assim como faz Teixeira (1998, p.12) é que a maioria dos pesquisadores da IA, desde o seu surgimento, planejavam:

[...] simular efetivamente processos mentais humanos e usar o computador para fundar uma ciência da mente. Para tanto, era preciso expandir a aplicação do modelo computacional para outros domínios além da simulação do raciocínio como acontecia com as máquinas de jogar xadrez ou demonstrar teoremas da lógica.

A IA trouxe a tona a problematização da natureza da mente e do próprio conceito de inteligência. Se a IA não conseguiu, satisfatoriamente, construir máquinas inteligentes, ela trouxe à baila a reflexão acerca do significado do 
conceito de inteligência e de outros conceitos amplamente empregados na Filosofia da Mente e na Ciência Cognitiva. O conceito de inteligência é basilar na Ciência Cognitiva e o modelo, nesse contexto, constitui uma ferramenta explicativa. Todavia, estudos contemporâneos apontam que o conceito de inteligência parece não ser satisfatoriamente contemplado na abordagem da Ciência Cognitiva tradicional. Nesse sentido, apresentaremos na seção seguinte a proposta de modelagem sistêmica.

\section{Modelos sistêmicos}

$\mathrm{Na}$ Ciência Cognitiva tradicional é considerado que são os estados mentais, enquanto representações da realidade, que causam o comportamento. Isso desencadearia a interpretação de que crenças e desejos são basicamente proposicionais, ou seja, o conhecimento comum é descritivo, pois descreve uma parte da realidade apreendida. Desse modo, admite-se que somos capazes de agir no mundo uma vez que possuímos representações deste e, como tais representações são internas, a cognição é interpretada conforme o modelo de mundo que temos acesso e segundo o qual raciocinamos e planejamos antes de agir (HASELAGER, 2004, p. 215-216). Em outras palavras, a hipótese da Ciência Cognitiva tradicional é de que os seres vivos representam estímulos recebidos do meio, criam representações internas do ambiente, consultam suas crenças e desejos, geram planejamentos e por fim elegem um planejamento para ser executado a fim de produzir um comportamento adequado. Tal postura ignora que também nos comportamos automaticamente e "racionaliza" o comportamento comum, considerando este tipo de comportamento como um processo de manipulação de símbolos.

A cognição, segundo a Ciência Cognitiva tradicional, se equipara ao processo de manipulação de símbolos de uma calculadora. Uma máquina de calcular pode produzir o símbolo ' 5 ', em resposta à entrada '2', '+', '3', estes símbolos não possuem significado para a máquina, mas para o usuário (aquele que inseriu a entrada) o resultado ' 5 ' "faz sentido" dado o significado que este usuário atribui aos símbolos (SHAPIRO, 2007, p.339). Tal visão aponta para a abordagem computacional da mente, cujas teses centrais apresentamos na primeira parte deste trabalho, segundo a qual o sistema nervoso de um ser vivo desempenha a mesma função de um hardware de computador (capta estímulos do meio e as representa de acordo com regras inatas ou aprendidas e por fim, desencadeia uma dada ação). A mente, nesse contexto, seria programas executados em hardwares cerebrais e "a cognição é separada do resto do mundo no sentido de que os processos cognitivos operam na 
liberdade simbólica dos órgãos dos sentidos 8 " (SHAPIRO, 2007, p.339, tradução nossa).

Como vimos, a mais relevante crítica que se faz ao modelo da cognição da Ciência Cognitiva tradicional diz respeito, justamente, aos projetos de sucesso serem baseados na manipulação de símbolos e, assim, facilmente descritos através de algoritmos (como jogar xadrez). O limite da modelagem se dá na tentativa de construir um modelo capaz de se movimentar em um ambiente desordenado não controlado, por exemplo. Esse tipo de atividade parece requerer capacidades muito mais simples do que a de jogar xadrez e mesmo assim, ainda se apresenta como um desafio para a Ciência Cognitiva tradicional. Em resposta à estas dificuldades, os pesquisadores da teoria da cognição situada e incorporada minimizam o papel das representações no processo de cognição.

Uma hipótese central que a teoria da cognição situada e incorporada defende é, além de considerar o ambiente na cognição, maximizar o papel do corpo na explicação da habilidades cognitivas. Segundo esta teoria, o processo de cognição pode emergir da interação dos atributos físicos dos corpos com o meio ambiente em que estes corpos estão situados. Para Clark: "A cognição não é um fenômeno que pode ser estudado com sucesso enquanto se marginaliza os papéis do corpo, do mundo e da ação ${ }^{9 "}$ (CLARK, 1999, p. 350, tradução nossa). As capacidades cognitivas humanas, então, dependem profundamente do entorno em que seus agentes epistêmicos estão inseridos. Todavia, o filósofo defende um externalismo ativo, destoando assim, da proposta externalista de Putnam (1975) e Burge (1979). Para Clark, o entorno exerce um poder causal no processo de conhecimento, e assim "Se eliminarmos o componente externo do sistema de condutas, é como se eliminarmos uma parte do próprio cérebro ${ }^{10 "}$ (CLARK, 1999, p. 13, tradução nossa). A tese de Clark diz respeito à hipótese de que os processos cognitivos podem ou não ocorrer dentro do cérebro. Tal tese aponta para o fato de que os fatores externos relevantes são ativos e que tem um papel crucial no momento presente e, dada a sua complementariedade com o organismo humano, acabam tendo um impacto direto no organismo e em sua conduta. Trata-se de estar situado em um ambiente e incorporar informações relevantes para um dado agente, que é ativo e não apenas representativo. Ao defender o externalismo ativo, Clark traz à baila uma explicação mais natural das ações e do comportamento inteligente.

Chemero (2007), por sua vez, considera que, uma vez os agentes situados em um ambiente, o papel da representação mental no desenvolvimento de habilidades é minimizado, pois "[...] uma parte da inteligência é 'carregada' do cérebro para o corpo e o ambiente ${ }^{11}$ " (CHEMERO, 2007, p. 181, tradução nossa). Nessa visão, os nossos corpos constituem 
ferramentas adequadas cujo design os torna fáceis de serem manipulados pelo cérebro. Por exemplo: nossos joelhos limitam os movimentos de nossas pernas, mas tornam o equilíbrio corporal e a locomoção possíveis. É através do corpo que conhecemos o ambiente e reconhecemos neste as possibilidades de nossas ações (CHEMERO, 2009, p.27). O corpo é importante, na abordagem de Chemero, pois é através dele que a mente pode fazer algo: o corpo, então, pode influenciar os processos mentais. Nessa perspectiva, o processo de aquisição e desenvolvimento de habilidades não é reduzido à manipulação de representações mentais. Os adeptos da Teoria da Cognição Incorporada e Situada defendem a hipótese segundo a qual existe uma dinâmica intrínseca entre os seres e seus ambientes (HASELAGER, 2004, p.221).

Os pressupostos da cognição situada e incorporada apontam para o fato de que nossas ações não são necessariamente e exclusivamente direcionadas pelas representações internas cerebrais, mas dependem também de processos dinâmicos estabelecidos entre o cérebro, o corpo e o ambiente. Tal processo é de natureza fluída, contínua e recorrente (HASELAGER, 2004, p. 224). Nesse sentido, apenas um modelo sistêmico poderia abarcar a complexidade dos processos cognitivos (dentre eles, a inteligência). Este necessitaria ser uma modelagem que abarca a complexidade e o conjunto de relações da mente, contextualizando-a no mundo real.

Nesse sentido, a problemática em relação à IA contemporânea consiste justamente na possibilidade de modelos computacionais estarem situados no meio ambiente e serem realmente capazes de adquirir e atualizar seus estados e funções de acordo com esse meio. Esse tipo de modelagem de inspiração externalista contemplaria o objetivo da IA ao modelar 0 comportamento inteligente.

\section{Considerações finais}

No presente trabalho procuramos analisar em que medida um modelo pode ser uma ferramenta explicativa para a abordagem do conceito de inteligência. Para tal, inicialmente focalizamos um problema clássico da filosofia, que é o de definir o conceito de inteligência: O que faz com que um sistema seja chamado de inteligente? Quais são os critérios que devem ser satisfeitos para isso? Estas interrogações ainda se fazem presentes e persistem nas problematizações e pesquisas filosóficas e cognitivistas. Muitas foram as tentativas de responder tais interrogações, a IA, por exemplo, enquanto um ramo da Ciência Cognitiva, propõe a análise da inteligência em termos de modelagem computacional. Nesse contexto, apontamos que o paradigma adotado pela IA tradicional não caracteriza satisfatoriamente a 
inteligência, embora proponha a caracterização mais viável tecnicamente para a modelagem (em termos de linguagem de programação e engenharia).

Em suma, procuramos mostrar que o paradigma funcional adotado pela IA tradicional, na medida em que desconsidera componentes contextuais e relacionais nos modelos cognitivos, não oferece bases para uma modelagem robótica capaz de ser contextualmente situada e de adquirir e aperfeiçoar habilidades. Sugerimos que uma modelagem pauta em um paradigma sistêmico (que compreende o modelo, o objeto e o ambiente, ou seja, uma complexidade mais geral do caso) poderia modelar uma característica como a inteligência de uma forma plausível. Por fim, tal tipo de modelagem requereria uma reestruturação técnica, em termos de linguagem de programação e de engenharia.

\section{Referências}

BOOLOS, G.S.; BURGESS, J.P.; JEFFREY, R.C. Computabilidade e lógica. São Paulo: Editora Unesp, 2012.

BURGE, T. Individualism and the mental. In: Midwest Studies in Philosophy 4, pp.73-121, 1979.

CHEMERO, A. Toward a situated, embodied realism. In: Center for the Ecological Study of Perception and Action, p.177-204, 2007.

Radical Embodied Cognitive Science. Cambridge: MIT Press, 2009.

CLARK, A. An embodied cognitive science? In: Trends in Cognitive Sciences, 3 p.345-351, 1999.

DESCARTES, R. Discurso do Método. São Paulo: Nova Cultural, 1987.

.Meditações metafisicas. São Paulo: Nova Cultural, 1988..

DUPUY, J. P. Nas origens das Ciências Cognitivas. Unesp: São Paulo, 1996.

HASELAGER, W.F.G. O Mal Estar do Representacionistmo: as sete dores de cabeça da Ciência Cognitiva. In: A.Ferreira, M.E.Q. Gonzalez, J.G.Coelho (orgs.). Encontro com as Ciências Cognitivas, v.4. São Paulo: Cultura Acadêmica, 2004. 
KHALFA, J. A natureza da inteligência. São Paulo: Editora Unesp, 1996.

MINSKY, M. Logical versus analogical or symbolic versus connectionist or neat versus scruffy. In: Al Magazine, vol. 12, no 2, pp. 34-51, 1991. Disponível em: $<$ https://www.aaai.org/ojs/index.php/aimagazine/article/view/894/812>. Acessado em: 10/01/2014.

(1995). Matter, mind and models. In: Proc. International Federation of Information Processing Congress, vol. 1, pp. 45-49, 1965. Disponível em: $<$ http://groups.csail.mit.edu/medg/people/doyle/gallery/minsky/mmm.html $>$. Acessado em 02/12/2013.

MURPHY, R.R. Introduction to Al Robotics. Cambridge: The MIT Press, 2000.

NEWELL, A.; SHAW, J. C.; SIMON, H. A.; Empirical explorations with the logic theory machine: A case study in heuristics. In: E.A. Feigenbaum \& J. Feldman (Eds.), Computers and thought. New York: McGraw-Hill, 1963.

PUTNAM, H. (1967) The nature of mental states. In: PUTNAM, H. Mind, language and reality.Cambridge: Cambridge University Press, 1975.

. The Meaning of a Meaning. In: Readings in language \& mind. Geirsson \& Losonsky (orgs), 1975.

SHAPIRO, L. The Embodied Research Program. In: Philosophy Compass 2, pp. 338-346, 2007. Disponível em: <http://portal.unifreiburg.de/cognition/lehre/archiv/ss13/matprosem/embodiedcognition/Shapiro EmbodiedCognition.pdf>. Acessado em: 24/02/2014.

STERNBERG, R. What's is Intelligence? Norwood, NJ: Ablex, 1986.

TASSINARI, R. \& D'OTTAVIANO, I.M.L. A Lógica e as Lógicas: Sobre a Noção de Sistema Formal e o Princípio da Liberdade Lógica. In: Informação, Conhecimento e Ação Ética. 1ed. Marília: Oficina Universitária/Cultura Acadêmica, p. 153-168, 2012.

TEIXEIRA, J. Mentes e Maquinas: Uma Introdução à Ciência Cognitiva. Porto Alegre: Editoras Artes Medicas, 1998.

TURING, A. Computing machinery and intelligence. Mind, 59, 1950. 


\footnotetext{
${ }^{1}$ Artificial Intelligence has no commonly accepted definitions. One the first textbooks on Al defined it as 'the study of ideas that enable computers to be intelligent,' which seemed to beg the question. A later textbook was more specific, 'Al is the attempt to get the computer to do things that, for the moment, people are better at'. This definition is interesting because it implies that once a task is performed successfully by computer, then the technique that made it possible is no longer $\mathrm{Al}$, but mundane.

2 We use the term model in this sense: To an observer $B$, an object $A^{*}$ is a model of an object $A$ to the extent that $B$ can use $A^{*}$ to answer questions that interest him about $A$.

${ }^{3}$ Em 1957, Newell, Shaw e Simon já haviam desenvolvido um programa chamado Logic Theoristic. Como aponta Dreyfus (1975, p.47), o Logic Theorist "utilizando uma busca heuristicamente orientada, na base do método de tentativas, provou 38 dentre 52 teoremas constantes do Principia Mathematica [de Alfred North Whitehead e Bertrand Arthur William Russell]. Dois anos depois, outro programa de Newell, Shaw e Simon, o Solucionador de Problemas em Geral (SPG), utilizando uma análise mais sofisticada de meios e fins, resolveu o problema dos 'canibais e os missionários' e outros problemas de complexidade semelhante.".

${ }^{4}[\ldots]$ specify them in detail, program them for digital computers, and study their behavior empirically by running them with a number of variations and under a variety of conditions.

5 The sentential calculus is a formalized system of mathematics, consisting of expressions built from combinations of basic symbols. Five of these expressions are taken as axioms, and there are rules of inference for generating new theorems from the axioms and from other theorems.

6 Como apontado por Tassinari \& D'Ottaviano (2012, p. 154) "Na sistematização de uma área do conhecimento, como as deduções sempre se apoiam em asserções anteriores, devemos aceitar determinadas asserções como primeiras para não cairmos em um regresso infinito; essas primeiras asserções, que aceitamos sem delas ter uma dedução, são chamadas de axiomas. A partir dos axiomas, regras de inferência estabelecem então como passar de uma asserção à outra, em deduções e demonstrações, gerando asserções chamadas de teoremas. Notemos que as regras de inferência também são argumentos válidos.".

7 By the nature of the methods, if the substitution method ever succeeds a single subproblem, the original theorem is proved.

${ }^{8}$ Cognition is cut off from the world in the sense that cognitive processes operate only on symbolic deliverances from the sense organs.

${ }^{9}$ Cognition is not a phenomenon that can be successfully studied while marginalizing the roles of body, world and action.

10 If we remove the external component the system's behavioral competence will drop, just as it would if we removed part of its brain.

11 [...] some of the intelligence is "off-loaded" from the brain to the body and environment.
} 